\title{
Enantioselective Effects of Metalaxyl Enantiomers on Breast Cancer Cells Metabolic Profiling Using HPLC-QTOF-Based Metabolomics
}

\author{
Ping Zhang ${ }^{1}$, Wentao Zhu ${ }^{2}$, Dezhen Wang ${ }^{2}$, Jin Yan ${ }^{2}$, Yao Wang ${ }^{2}$ and Lin He ${ }^{1, *}$ \\ 1 College of Plant Protection, Southwest University, Chongqing 400715, China; pingz@swu.edu.cn \\ 2 Beijing Advanced Innovation Center for Food Nutrition and Human Health, \\ Department of Applied Chemistry, China Agricultural University, Beijing 100193, China; \\ wentaozhu@cau.edu.cn (W.Z.); wangdezhen@cau.edu.cn (D.W.); ping17028@gmail.com (J.Y.); \\ yaow@cau.edu.cn (Y.W.) \\ * Correspondence: helinok@vip.tom.com; Tel.: +86-23-6825-1541; Fax: +86-23-6825-1514
}

Academic Editor: William Chi-shing Cho

Received: 6 December 2016; Accepted: 4 January 2017; Published: 12 January 2017

\begin{abstract}
In this study, an integrative high-performance liquid chromatography coupled with quadrupole time-of-flight tandem mass spectrometry (HPLC-QTOF) based metabolomics approach was performed to evaluate the enantioselective metabolic perturbations in MCF-7 cells after treatment with $R$-metalaxyl and $S$-metalaxyl, respectively. Untargeted metabolomics profile, multivariate pattern recognition, metabolites identification, and pathway analysis were determined after metalaxyl enantiomer exposure. Principal component analysis (PCA) and partitial least-squares discriminant analysis (PLS-DA) directly reflected the enantioselective metabolic perturbations induced by metalaxyl enantiomers. On the basis of multivariate statistical results, a total of 49 metabolites including carbohydrates, amino acids, nucleotides, fatty acids, organic acids, phospholipids, indoles, derivatives, etc. were found to be the most significantly changed metabolites and metabolic fluctuations caused by the same concentration of $R$-metalaxyl and $S$-metalaxyl were enantioselective. Pathway analysis indicated that $R$-metalaxyl and $S$-metalaxyl mainly affected the 7 and 10 pathways in MCF-7 cells, respectively, implying the perturbed pathways induced by metalaxyl enantiomers were also enantioselective. Furthermore, the significantly perturbed metabolic pathways were highly related to energy metabolism, amino acid metabolism, lipid metabolism, and antioxidant defense. Such results provide more specific insights into the enantioselective metabolic effects of chiral pesticides in breast cancer progression, reveal the underlying mechanisms, and provide available data for the health risk assessments of chiral environmental pollutants at the molecular level.
\end{abstract}

Keywords: metalaxyl; metabolomics; enantiomers; HPLC-QTOF; metabolic perturbations

\section{Introduction}

The extensive application of pesticides in agriculture, individual households, and in public spaces leaves pervasive residue in the environment and exposes both humans and animals to direct and indirect sources, including via food consumption; lawn, garden, and household use; and occupational exposure [1,2]. Several pesticides are known to be associated with cancer development in experimental and epidemiological studies of farmers as well as pesticide manufacturing workers [3-6]. Although the mechanisms of cancer development induced by pesticide exposure are not clear, some potential mechanisms are DNA damage, immune response abnormality, oxidative stress chronic inflammation, and chromosome aberration [7-10]. Given the widespread applications and pervasive residue associated with pesticides, there is a need to be vigilant in surveillance of potential cancer impacts on humans. 
Metalaxyl [N-(2,6-dimethylphenyl)- $N$-(methoxyacetyl)-D,L-alaninemethylester, 57837-19-1] is a systemic fungicide with curative actions against oomycetes and water mold fungi in plants [11]. Its effectiveness comes from specific inhibition of RNA polymerase-1 activity and uridine incorporation into RNA process [12]. Given its broad spectrum of activity, metalaxyl is not only used on food crops but also on non-food and residential crops including tobacco, trees, ornamental plants, and lawns. Metalaxyl has an asymmetric carbon atom and consists of two enantiomers, which possess similar physicochemical properties in non-chiral environments and different activities in biological systems (Figure 1) [13]. Although metalaxyl is classified as a low-toxicity pesticide, it is mobile, persistent, and readily leached in soils. Metalaxyl residue was detected in groundwater, which poses a great threat to human and animal health [9]. Studies demonstrated cytogenetic effects of metalaxyl on human and animal chromosomes in vitro [14]. Furthermore, cocarcinogenic potential and nephrotoxicity were also reported in mice $[15,16]$.

A
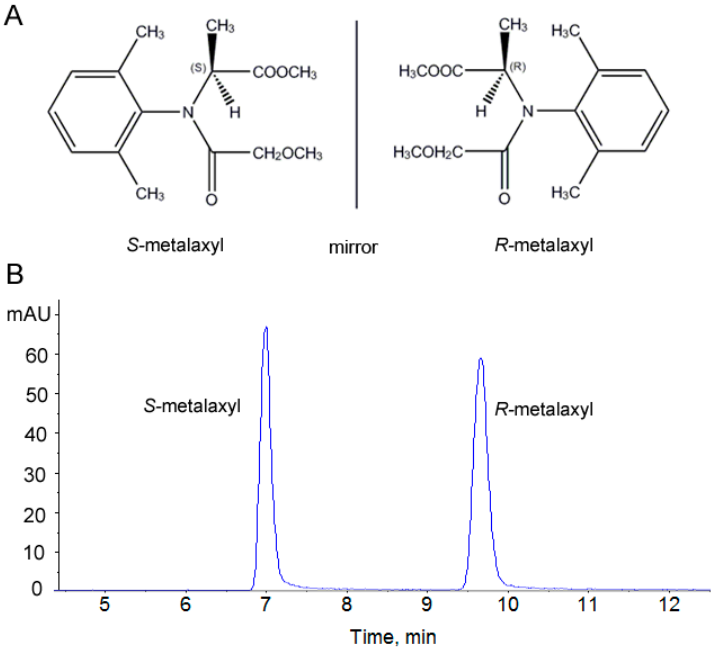

Figure 1. Chemical structure (A) and representative chromatogram (B) of metalaxyl enantiomers.

Breast cancer is one of the most common cancers in women and the American Cancer Society estimates that in 2016 breast cancer will have accounted for $29 \%$ of all new cancer cases diagnosed in women in the USA $[17,18]$. It is widely recognized that the development of cancer is the combined results of genetic predisposition and environmental factors. There is considerable evidence to show that cumulative and sustained estrogen exposure is a key promoter of breast tumor proliferation [19]. Although metalaxyl is not classified as an estrogen disrupter, studies have demonstrated that it has cytotoxicity, nephrotoxicity, cell transformation, and cocarcinogenic activity in mice [20-22]. Furthermore, metalaxyl has the ability to activate human pregnane $\mathrm{X}$ receptor, which is highly related to breast cancer cell metabolism [23]. Human pregnane $X$ receptor (hPXR) is a primary transcription factor of CYP3A4 and an efflux transporter of multi-drug resistance gene (MDR1). Activation of hPXR will lead to upregulated expression of CYP3A4 and MDR1, which are two possible mediators of hPXR-related drug resistance in breast cancer [24]. Thus metalaxyl exposure may significantly affect the metabolome of MCF-7 cells through activating hPXR.

To further understand biological systems' response to metalaxyl enantiomers exposure, a metabolomics profile was adopted to create a full picture of cell metabolic perturbations. Metabolomics is an important branch in the area of "omics" research, which is defined as the systematic study of multiparametric metabolic responses of organisms to perturbations such as diseases, environmental factors, genetic variations, and other stimuli [25-27]. Over the past decades, there has been an increased interest in cellular metabolism, which is regarded as a possible means of cancer treatment, especially after the vital finding of upregulated glucose consumption and lactate production in cancer cells under aerobic conditions by Otto Warburg in the 1930s [28]. Altered metabolism 
helps cancer cells sustain a higher proliferation rate even in suboptimal environments, resist certain cell signals, and also prevent immune response. Therefore, alterations in cellular metabolism are expected as one of the vital hallmarks in cancer development [29]. Some major metabolic alterations including enhanced fatty acids synthesis, upregulated utilization of the pentose phosphate pathway, increased production of lactate, altered utilization of the tricarboxylic acid (TCA) cycle, and reduced transport of pyruvate into mitochondria were identified in cancer cells [30]. Therefore, the emerging field of metabolomics may provide insights into integrated perturbed metabolic profile in cancer research and help us find key biomarkers, which may act as a reliable clinical tool for cancer diagnostic screening and therapy [31].

The aim of this study was to evaluate the enantioselective metabolic perturbations induced by metalaxyl enantiomers in MCF-7 cells using a high-performance liquid chromatography coupled with quadrupole time-of-flight tandem mass spectrometry (HPLC-QTOF) based untargeted metabolomics approach. HPLC-QTOF based metabolomics profile, multivariate pattern recognition, metabolites identification, and pathway analysis were determined in MCF-7 cells after R-metalaxyl and S-metalaxyl treatments, respectively. To our best knowledge, this is the first enantioselective study of metabolic alterations induced by metalaxyl enantiomers in breast cancer cells using metabolomics techniques. Such results may contribute to explaining the enantioselective metabolic and toxic effects of chiral pesticides in breast cancer progression, revealing the underlying mechanisms, and providing available data for health risk assessments of chiral environmental pollutants.

\section{Results and Discussion}

\subsection{Assessment of the Stability and Reproducibility of the HPLC-QTOF Method}

Quality control (QC) samples were obtained by pooling metalaxyl enantiomer-treated cell samples and prepared using the same protocol as sample preparation. For the stability of HPLC-QTOF system, QC sample was injected randomly during the sequence analysis. As for the reproducibility of the sample preparation, $1000 \mu \mathrm{L}$ of a mixed control sample was split into five parts with $200 \mu \mathrm{L}$ each and treated with the same preparation protocol. A blank sample (ultrapure water), prepared in the same way as the other samples, was injected every five samples to minimize the carry-over between sample analyses.

The results for system stability and preparation method reproducibility are shown in Figure S1. We examined coefficient of variation $(\mathrm{CV})$ values of 1061 mass variables that were detected in $70 \%$ of QC data. As shown in Figure S1, more than $90 \%$ of QTOF variables had CV $<20 \%$, and $<4 \%$ had CV $>30 \%$, indicating excellent system stability. For reproducibility, more than $80 \%$ of QTOF variables had $\mathrm{CV}<20 \%$, and $<7 \%$ had CV $>30 \%$, which also implied good reproducibility. Such results indicate that the established analytical method is highly stable and reproducible, and could be employed to analyze large-scale cell samples in metabolomics experiments.

\subsection{Multivariate Pattern Recognition Analysis}

In order to investigate the global metabolites changes in MCF-7 cells after metalaxyl enantiomer exposure, Principal component analysis (PCA) was employed to analyze the QTOF data set first. PCA is an unsupervised multivariate data analysis method often adopted to visualize grouping trends and outliers in data. Principle component 1 (PC1) versus component 2 (PC1) of MCF-7 cells in positive, negative, and both modes are shown in Figure 2. Good separation between metalaxyl enantiomer-treated groups and the control group were displayed in PC1 and PC2 plots of PCA, suggesting that metalaxyl enantiomers significantly disturbed the MCF-7 cell metabolome after seven days of consecutive exposure. The fitness and prediction capabilities of PCA modes were evaluated by $R^{2} X$ (cum) and $Q^{2}$ (cum), respectively. Figure 2 also showed $Q^{2} X($ cum $)$ and $Q^{2}$ (cum) of PCA in different modes. $Q^{2}$ in the positive, negative, and both modes were all over 0.5 , displaying the excellent predictive capability. In addition, metabolome changes induced by $R$-metalaxyl and $S$-metalaxyl were also clearly separated, which means $R$-metalaxyl and $S$-metalaxyl have enantioselective effects 
on MCF-7 cell metabolite profiling. Moreover, cells treated with a single enantiomer at different concentrations $(10$ and $50 \mu \mathrm{M})$ were clearly separated, indicating that the metabolome change attributable to different treated concentrations was also different. To further identify the metabolites that account for the PCA separation, supervised PLS-DA was employed to construct the mode based on HPLC-QTOF ion peak areas of cell metabolites. Representative PLS-DA plots of treated groups and the control group are shown in Figure 3. $R$-metalaxyl- and S-metalaxyl-treated groups are clearly separated with the control group. Moreover, the $R$-metalaxyl-treated group and $S$-metalaxyl-treated group also displayed a clear separation, which means the metabolite changes induced by the two enantiomers were enantioselective. Generally, $R^{2} X, R^{2} Y$, and $Q^{2} Y$ were adopted to assess the quality of the PLS-DA mode. Without a high $R^{2} Y$, it is impossible to obtain a high $Q^{2} Y$ and a robust model was linked to a $Q^{2}>0.4$ [32]. As shown in Figure 3, the parameters of $R^{2} X, R^{2} Y$, and $Q^{2} Y$ were acceptable for single negative and positive mode datasets, and the combination of two datasets also displayed an excellent prediction, with $R^{2} Y=0.986$ and $Q^{2} Y=0.948$.
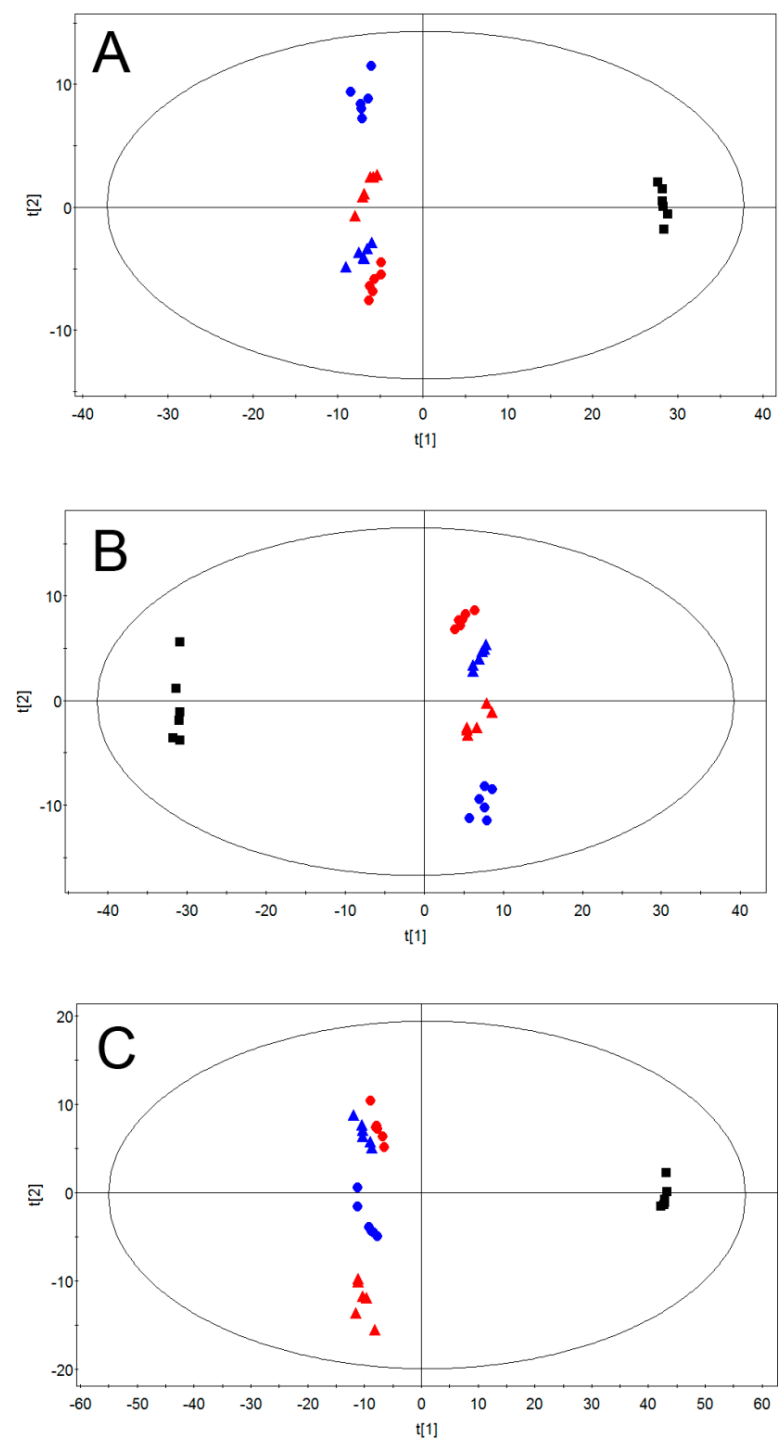

Figure 2. PCA score plots based on QTOF data sets of MCF-7 cells treated with different concentrations of metalaxyl enantiomers. $\mathbf{D}$ Control, $\boldsymbol{\Delta} S$-metalaxyl $(10 \mu \mathrm{M}), \boldsymbol{\Delta} S$-metalaxyl $(50 \mu \mathrm{M}), \bullet R$-metalaxyl $(10 \mu \mathrm{M}), \bullet R$-metalaxyl (50 $\mu \mathrm{M})$. (A) Positive mode, $\left(R^{2} X=0.656, Q^{2}=0.552\right) ;(\mathbf{B})$ negative mode, $\left(R^{2} X=0.715, Q^{2}=0.629\right) ;(\mathbf{C})$ negative + positive mode, $\left(R^{2} X=0.783, Q^{2}=0.685\right)$. 

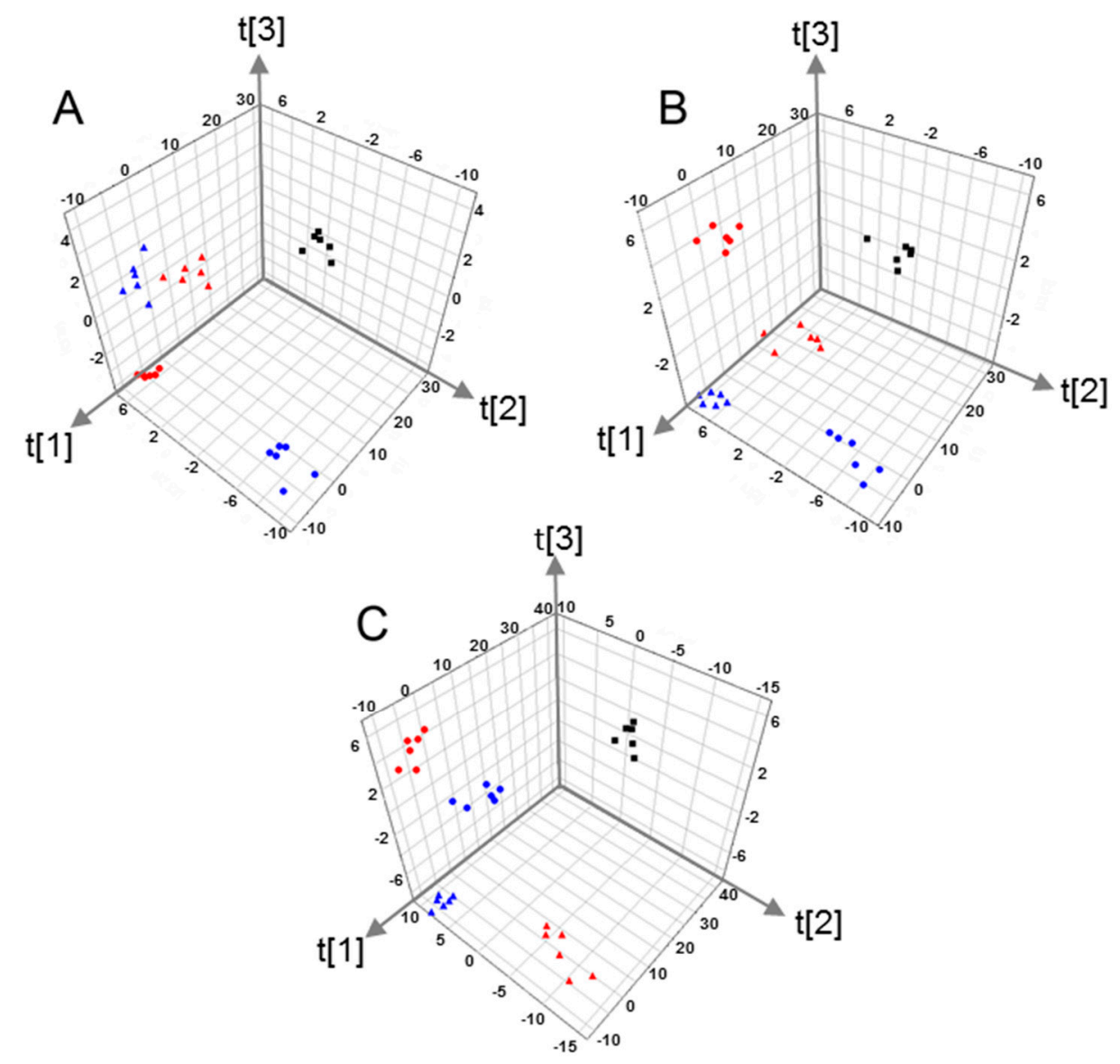

Figure 3. The PLS-DA score plots based on QTOF data sets of MCF-7 cells treated with different concentration of metalaxyl enantiomers. Control, $\Delta S$-metalaxyl $(10 \mu \mathrm{M}), \Delta S$-metalaxyl $(50 \mu \mathrm{M})$, - $R$-metalaxyl $(10 \mu \mathrm{M}), \Delta R$-metalaxyl $(50 \mu \mathrm{M})$. (A) Positive mode, $\left(R^{2} X=0.829, R^{2} Y=0.982, Q^{2}=0.913\right)$; (B) negative mode, $\left(R^{2} X=0.816, R^{2} Y=0.99, Q^{2}=0.957\right)$; (C) negative + positive mode, $\left(R^{2} X=0.803\right.$, $\left.R^{2} Y=0.986, Q^{2}=0.948\right)$.

\subsection{Metabolite Identification}

To determine the metabolites that are responsible for metalaxyl enantiomer intervention, each $R$-metalaxyl- and S-metalaxyl-treated group was compared with a control group by PLS-DA analysis. The variable importance in the project (VIP) value derived from the PLS-DA mode is an important parameter for each independent variable. Higher VIP scores are considered more relevant in classification. In this study, the VIP value of each peak was calculated to identify its contribution to the classification. On the basis of the VIP threshold (VIP $>1)$ and the student's $t$ test $p$ value $(p<0.05)$, a total of 49 metabolites ( 35 from the negative mode and 14 from the positive mode) were finally identified (Table 1). These significant changed metabolites were amino acids, nucleotides, fatty acids, carbohydrates, phospholipids, indoles, derivatives, and so on. $R$-metalaxyl- and $S$-metalaxyl-treated $(10 \mu \mathrm{M})$ groups induced 27 and 36 endogenous metabolite increases, and five and eight metabolites decreased, respectively. High concentration $(50 \mu \mathrm{M})$ caused 29 and 35 endogenous metabolites to be upregulated, and six and eight metabolites to be downregulated, respectively. Such results indicated that metabolic profiling changes caused by the same concentration of $R$-metalaxyl and $S$-metalaxyl were enantioselective. Furthermore, some metabolites changed with the treated concentration, such as histidine, lactate, glucose, alanine, succinate, citrate, and arginine (Figure 4). 
Table 1. Significant changed metabolites induced by R-metalaxyl and S-metalaxyl in MCF-7 cells. C, Control group; L, Low-dose group; H, High-dose group.

\begin{tabular}{|c|c|c|c|c|c|c|c|c|c|c|c|c|c|c|c|c|}
\hline \multirow{2}{*}{ No. } & \multirow{2}{*}{ Mode } & \multirow{2}{*}{ Compounds } & \multirow{2}{*}{ Mass } & \multicolumn{3}{|c|}{ S-Metalaxyl L vs. C } & \multicolumn{3}{|c|}{ S-Metalaxyl H vs. C } & \multicolumn{3}{|c|}{ R-Metalaxyl L vs. C } & \multicolumn{3}{|c|}{ R-Metalaxyl H vs. C } & \multirow{2}{*}{ Class } \\
\hline & & & & VIP $^{a}$ & $\mathrm{FC}^{\mathrm{b}}$ & Trend $^{c}$ & VIP & FC & Trend & VIP & FC & Trend & VIP & FC & Trend & \\
\hline 1 & Negative & Lactate & 72.0213 & 1.14 & 3.55 & $\uparrow$ & $<1$ & 2.80 & $\uparrow$ & $<1$ & 2.28 & $\uparrow$ & $<1$ & 1.66 & $\uparrow$ & Hydroxy acids and derivatives \\
\hline 2 & Negative & Propionate & 74.0368 & 1.17 & 1.92 & $\uparrow$ & $<1$ & 2.22 & $\uparrow$ & 1.13 & 1.42 & $\uparrow$ & 1.09 & 1.38 & $\uparrow$ & Carboxylic acids and derivatives \\
\hline 3 & Negative & Glycine & 75.0322 & 1.18 & 3.64 & $\uparrow$ & 1.16 & 3.55 & $\uparrow$ & 1.17 & 3.44 & $\uparrow$ & 1.19 & 3.91 & $\uparrow$ & Carboxylic acids and derivatives \\
\hline 4 & Negative & Alanine & 89.0474 & 1.17 & 2.19 & $\uparrow$ & 1.16 & 2.53 & $\uparrow$ & 1.17 & 2.30 & $\uparrow$ & 1.19 & 2.72 & $\uparrow$ & Carboxylic acids and derivatives \\
\hline 5 & Negative & Serine & 105.0420 & 1.15 & 1.62 & $\uparrow$ & 1.13 & 1.62 & $\uparrow$ & 1.11 & 1.49 & $\uparrow$ & 1.17 & 1.73 & $\uparrow$ & Carboxylic acids and derivatives \\
\hline 6 & Negative & Proline & 115.0624 & 1.23 & 1.21 & $\uparrow$ & 1.57 & 1.26 & $\uparrow$ & $<1$ & 1.29 & $\uparrow$ & 1.06 & 1.20 & $\uparrow$ & Carboxylic acids and derivatives \\
\hline 7 & Negative & Guanidineacetate & 117.0419 & 1.18 & 2.55 & $\uparrow$ & 1.16 & 2.45 & $\uparrow$ & 1.18 & 2.22 & $\uparrow$ & 1.19 & 2.75 & $\uparrow$ & Carboxylic acids and derivatives \\
\hline 8 & Negative & Succinate & 118.0259 & 1.18 & 1.85 & $\uparrow$ & 1.15 & 1.60 & $\uparrow$ & 1.12 & 1.34 & $\uparrow$ & 1.08 & 1.24 & $\uparrow$ & Carboxylic acids and derivatives \\
\hline 9 & Negative & Glutaconate & 130.0256 & 1.16 & 2.11 & $\uparrow$ & 1.12 & 2.12 & $\uparrow$ & 1.15 & 1.96 & $\uparrow$ & 1.12 & 2.17 & $\uparrow$ & Carboxylic acids and derivatives \\
\hline 10 & Negative & 3-Methyl-2-oxovalerate & 130.0623 & $<1$ & 3.10 & $\uparrow$ & 1.03 & 4.01 & $\uparrow$ & $<1$ & 3.93 & $\uparrow$ & $<1$ & 3.53 & $\uparrow$ & Keto acids and derivatives \\
\hline 11 & Negative & Creatine & 131.0582 & 1.17 & 2.76 & $\uparrow$ & 1.16 & 3.03 & $\uparrow$ & 1.17 & 3.45 & $\uparrow$ & 1.19 & 3.05 & $\uparrow$ & Carboxylic acids and derivatives \\
\hline 12 & Negative & Aspartate & 133.0371 & 1.21 & 2.14 & $\uparrow$ & 1.23 & 2.24 & $\uparrow$ & $<1$ & 2.25 & $\uparrow$ & $<1$ & 2.47 & $\uparrow$ & Carboxylic acids and derivatives \\
\hline 13 & Negative & Muconate & 142.0237 & 3.50 & 0.82 & $\downarrow$ & 4.12 & 0.75 & $\downarrow$ & 4.9 & 0.70 & $\downarrow$ & 4.1 & 0.76 & $\downarrow$ & Fatty acids and conjugates \\
\hline 14 & Negative & Glutamine & 146.0685 & 1.66 & 4.84 & $\uparrow$ & 1.62 & 4.87 & $\uparrow$ & $<1$ & 5.10 & $\uparrow$ & $<1$ & 5.34 & $\uparrow$ & Carboxylic acids and derivatives \\
\hline 15 & Negative & Glutamate & 147.0524 & 1.14 & 0.67 & $\downarrow$ & 1.14 & 0.65 & $\downarrow$ & 1.15 & 0.59 & $\downarrow$ & 1.16 & 0.66 & $\downarrow$ & Carboxylic acids and derivatives \\
\hline 16 & Negative & Methionine & 149.0503 & 1.12 & 2.52 & $\uparrow$ & 1.01 & 2.32 & $\uparrow$ & $<1$ & 1.56 & $\uparrow$ & 1.12 & 2.54 & $\uparrow$ & Carboxylic acids and derivatives \\
\hline 17 & Negative & 4-Imidazolone-5-propionate & 156.0523 & 1.15 & 3.19 & $\uparrow$ & 1.16 & 4.79 & $\uparrow$ & 1.14 & 4.65 & $\uparrow$ & 1.14 & 2.84 & $\uparrow$ & Imidazoles \\
\hline 18 & Negative & 2-Aminomuconate & 157.0353 & 1.12 & 0.58 & $\downarrow$ & 1.13 & 0.42 & $\downarrow$ & 1.14 & 0.46 & $\downarrow$ & 1.14 & 0.44 & $\downarrow$ & Carboxylic acids and derivatives \\
\hline 19 & Negative & Benzoate & 157.9976 & 4.06 & 1.81 & $\uparrow$ & 3.71 & 1.72 & $\uparrow$ & 3.65 & 1.80 & $\uparrow$ & 3.39 & 1.71 & $\uparrow$ & $\begin{array}{c}\text { Benzene and substituted } \\
\text { derivatives }\end{array}$ \\
\hline 20 & Negative & 3-Phosphonopyruvate & 167.9816 & 1.10 & 1.88 & $\uparrow$ & $<1$ & 1.33 & $\uparrow$ & 1.06 & 1.42 & $\uparrow$ & 1.05 & 1.87 & $\uparrow$ & $\begin{array}{l}\text { Organic phosphoric acids } \\
\text { and derivatives }\end{array}$ \\
\hline 21 & Negative & Glyceraldehyde-3-phosphate & 169.9970 & 1.14 & 0.05 & $\downarrow$ & 1.13 & 0.08 & $\downarrow$ & 1.15 & 0.09 & $\downarrow$ & 1.13 & 0.14 & $\downarrow$ & $\begin{array}{l}\text { Carbohydrates and } \\
\text { carbohydrate conjugates }\end{array}$ \\
\hline 22 & Negative & Aconitate & 174.0157 & 1.94 & 1.93 & $\uparrow$ & 1.25 & 1.77 & $\uparrow$ & $<1$ & 1.91 & $\uparrow$ & $<1$ & 1.86 & $\uparrow$ & Carboxylic acids and derivatives \\
\hline 23 & Negative & $\mathrm{N}$-acetylaspartate & 175.0478 & 2.36 & 3.78 & $\uparrow$ & 2.35 & 3.93 & $\uparrow$ & 1.28 & 4.62 & $\uparrow$ & 1.39 & 3.94 & $\uparrow$ & Carboxylic acids and derivatives \\
\hline 24 & Negative & Glucose & 180.0629 & 1.98 & 2.15 & $\uparrow$ & 1.71 & 1.92 & $\uparrow$ & 1.11 & 2.45 & $\uparrow$ & $<1$ & 2.01 & $\uparrow$ & $\begin{array}{l}\text { Carbohydrates and } \\
\text { carbohydrate conjugates }\end{array}$ \\
\hline 25 & Negative & Tyrosine & 181.0728 & 1.18 & 2.68 & $\uparrow$ & 1.16 & 2.97 & $\uparrow$ & 1.17 & 2.80 & $\uparrow$ & 1.18 & 2.86 & $\uparrow$ & Phenylpropanoic acids \\
\hline 26 & Negative & $\mathrm{N}$-Acetyl-L-glutar & 189.0632 & 1.76 & 0.11 & $\downarrow$ & 1.71 & 0.13 & $\downarrow$ & $<1$ & 0.09 & $\downarrow$ & $<1$ & 0.16 & $\downarrow$ & Carboxylic acids and derivatives \\
\hline 27 & Negative & 3-Dehydroquinate & 190.0404 & $<1$ & 2.64 & $\uparrow$ & 1.22 & 4.38 & $\uparrow$ & $<1$ & 4.33 & $\uparrow$ & $<1$ & 2.37 & $\uparrow$ & Alcohols and polyols \\
\hline 28 & Negative & Citrate & 192.0268 & 3.26 & 7.09 & $\uparrow$ & 3.56 & 8.97 & $\uparrow$ & 2.31 & 6.23 & $\uparrow$ & 2.98 & 8.37 & $\uparrow$ & Carboxylic acids and derivatives \\
\hline 29 & Negative & Galactonate & 196.0571 & 1.14 & 0.24 & $\downarrow$ & 1.12 & 0.14 & $\downarrow$ & 1.13 & 0.24 & $\downarrow$ & 1.08 & 0.25 & $\downarrow$ & Hydroxy acids and derivatives \\
\hline 30 & Negative & Pantothenate & 219.1099 & 2.04 & 11.52 & $\uparrow$ & 1.97 & 11.39 & $\uparrow$ & 1.07 & 13.02 & $\uparrow$ & $<1$ & 10.04 & $\uparrow$ & Carboxylic acids and derivatives \\
\hline 31 & Negative & $N$-Acetyl-D-glucosamine & 257.0662 & 1.07 & 2.29 & $\uparrow$ & 1.03 & 2.27 & $\uparrow$ & $<1$ & 2.01 & $\uparrow$ & $<1$ & 2.41 & $\uparrow$ & $\begin{array}{l}\text { Carbohydrates and } \\
\text { carbohydrate conjugates }\end{array}$ \\
\hline 32 & Negative & Acetylcarnosine & 306.0308 & $<1$ & 0.95 & $\downarrow$ & 1.66 & 0.82 & $\downarrow$ & $<1$ & 0.77 & $\downarrow$ & $<1$ & 0.91 & $\downarrow$ & Carboxylic acids and derivatives \\
\hline 33 & Negative & dUMP & 344.0442 & 1.24 & 0.73 & $\downarrow$ & 1.1 & 0.76 & $\downarrow$ & $<1$ & 0.76 & $\downarrow$ & $<1$ & 0.72 & $\downarrow$ & Pyrimidine nucleotides \\
\hline 34 & Negative & dGDP & 427.0289 & 1.12 & 3.95 & $\uparrow$ & 1.11 & 4.07 & $\uparrow$ & $<1$ & 4.16 & $\uparrow$ & $<1$ & 3.91 & $\uparrow$ & Purine nucleotides \\
\hline 35 & Negative & dGTP & 506.9954 & 1.95 & 2.51 & $\uparrow$ & 1.95 & 2.60 & $\uparrow$ & $<1$ & 2.37 & $\uparrow$ & $<1$ & 2.56 & $\uparrow$ & Purine nucleotides \\
\hline
\end{tabular}


Table 1. Cont.

\begin{tabular}{|c|c|c|c|c|c|c|c|c|c|c|c|c|c|c|c|c|}
\hline \multirow{2}{*}{ No. } & \multirow{2}{*}{ Mode } & \multirow{2}{*}{ Compounds } & \multirow{2}{*}{ Mass } & \multicolumn{3}{|c|}{ S-Metalaxyl L vs. C } & \multicolumn{3}{|c|}{ S-Metalaxyl H vs. C } & \multicolumn{3}{|c|}{ R-Metalaxyl L vs. C } & \multicolumn{3}{|c|}{ R-Metalaxyl H vs. C } & \multirow{2}{*}{ Class } \\
\hline & & & & VIP ${ }^{a}$ & $\mathrm{FC}^{\mathrm{b}}$ & Trend $^{\mathrm{c}}$ & VIP & FC & Trend & VIP & FC & Trend & VIP & FC & Trend & \\
\hline 36 & Positive & Valine & 117.0779 & 1.27 & 1.57 & $\uparrow$ & 1.15 & 1.43 & $\uparrow$ & 1.23 & 1.41 & $\uparrow$ & 1.06 & 1.50 & $\uparrow$ & Carboxylic acids and derivatives \\
\hline 37 & Positive & Pyroglutamate & 129.0416 & 1.08 & 0.64 & $\downarrow$ & $<1$ & 0.81 & $\downarrow$ & $<1$ & 0.87 & $\downarrow$ & 1.11 & 0.56 & $\downarrow$ & Carboxylic acids and derivatives \\
\hline 38 & Positive & 3-Hydroxy-L-proline & 131.0677 & 1.28 & 7.63 & $\uparrow$ & 1.41 & 8.39 & $\uparrow$ & 1.41 & 8.44 & $\uparrow$ & 1.26 & 7.58 & $\uparrow$ & Carboxylic acids and derivatives \\
\hline 39 & Positive & Leucine/Isoleucine & 131.0932 & 1.82 & 2.98 & $\uparrow$ & 1.72 & 2.78 & $\uparrow$ & 1.33 & 2.48 & $\uparrow$ & 1.81 & 2.87 & $\uparrow$ & Carboxylic acids and derivatives \\
\hline 40 & Positive & Glutarate & 132.0521 & 1.07 & 3.59 & $\uparrow$ & 1.07 & 3.61 & $\uparrow$ & 1.04 & 3.60 & $\uparrow$ & 1.11 & 3.68 & $\uparrow$ & Carboxylic acids and derivatives \\
\hline 41 & Positive & Histidine & 155.0674 & $<1$ & 3.47 & $\uparrow$ & 1.04 & 4.96 & $\uparrow$ & $<1$ & 3.07 & $\uparrow$ & 1.06 & 4.94 & $\uparrow$ & Carboxylic acids and derivatives \\
\hline 42 & Positive & Phenylalanine & 165.0773 & 1.43 & 2.05 & $\uparrow$ & 1.23 & 1.77 & $\uparrow$ & 1.07 & 1.61 & $\uparrow$ & 1.56 & 2.18 & $\uparrow$ & Phenylpropanoic acids \\
\hline 43 & Positive & Arginine & 174.1099 & 2.13 & 6.52 & $\uparrow$ & 1.86 & 5.83 & $\uparrow$ & 2.3 & 6.99 & $\uparrow$ & 1.75 & 5.55 & $\uparrow$ & Carboxylic acids and derivatives \\
\hline 44 & Positive & 3-Indolebutyrate & 203.1137 & 1.75 & 5.98 & $\uparrow$ & 1.79 & 6.25 & $\uparrow$ & 1.83 & 6.68 & $\uparrow$ & 1.7 & 5.51 & $\uparrow$ & Indoles and derivatives \\
\hline 45 & Positive & Tryptophan & 204.0874 & 1.07 & 2.41 & $\uparrow$ & $<1$ & 2.23 & $\uparrow$ & $<1$ & 1.91 & $\uparrow$ & 1.2 & 2.68 & $\uparrow$ & Indoles and derivatives \\
\hline 46 & Positive & Propionyl-L-carnitine & 217.1288 & $<1$ & 1.50 & $\uparrow$ & $<1$ & 1.44 & $\uparrow$ & 1.1 & 1.72 & $\uparrow$ & 1.02 & 1.57 & $\uparrow$ & Fatty acid esters \\
\hline 47 & Positive & Glycerophosphocholine & 257.1003 & 1.98 & 10.24 & $\uparrow$ & 1.98 & 10.24 & $\uparrow$ & 2.29 & 11.71 & $\uparrow$ & 2.08 & 10.77 & $\uparrow$ & Glycerophospholipids \\
\hline 48 & Positive & Glutathione & 307.0812 & 3.54 & 1.98 & $\uparrow$ & 3.49 & 1.97 & $\uparrow$ & 3.63 & 2.01 & $\uparrow$ & 3.36 & 1.94 & $\uparrow$ & Carboxylic acids and derivatives \\
\hline 49 & Positive & UDP- $N$-acetylglucosamine & 607.0766 & 1.25 & 4.12 & $\uparrow$ & 1.23 & 4.07 & $\uparrow$ & 1.21 & 4.03 & $\uparrow$ & 1.21 & 4.04 & $\uparrow$ & Pyrimidine nucleotides \\
\hline
\end{tabular}

${ }^{a}$ VIP represents variable importance in the project value derived from PLS-DA mode; ${ }^{b}$ FC represents fold change; ${ }^{c} \uparrow$ represents up-regulated trend and $\downarrow$ represents down-regulated trend. 


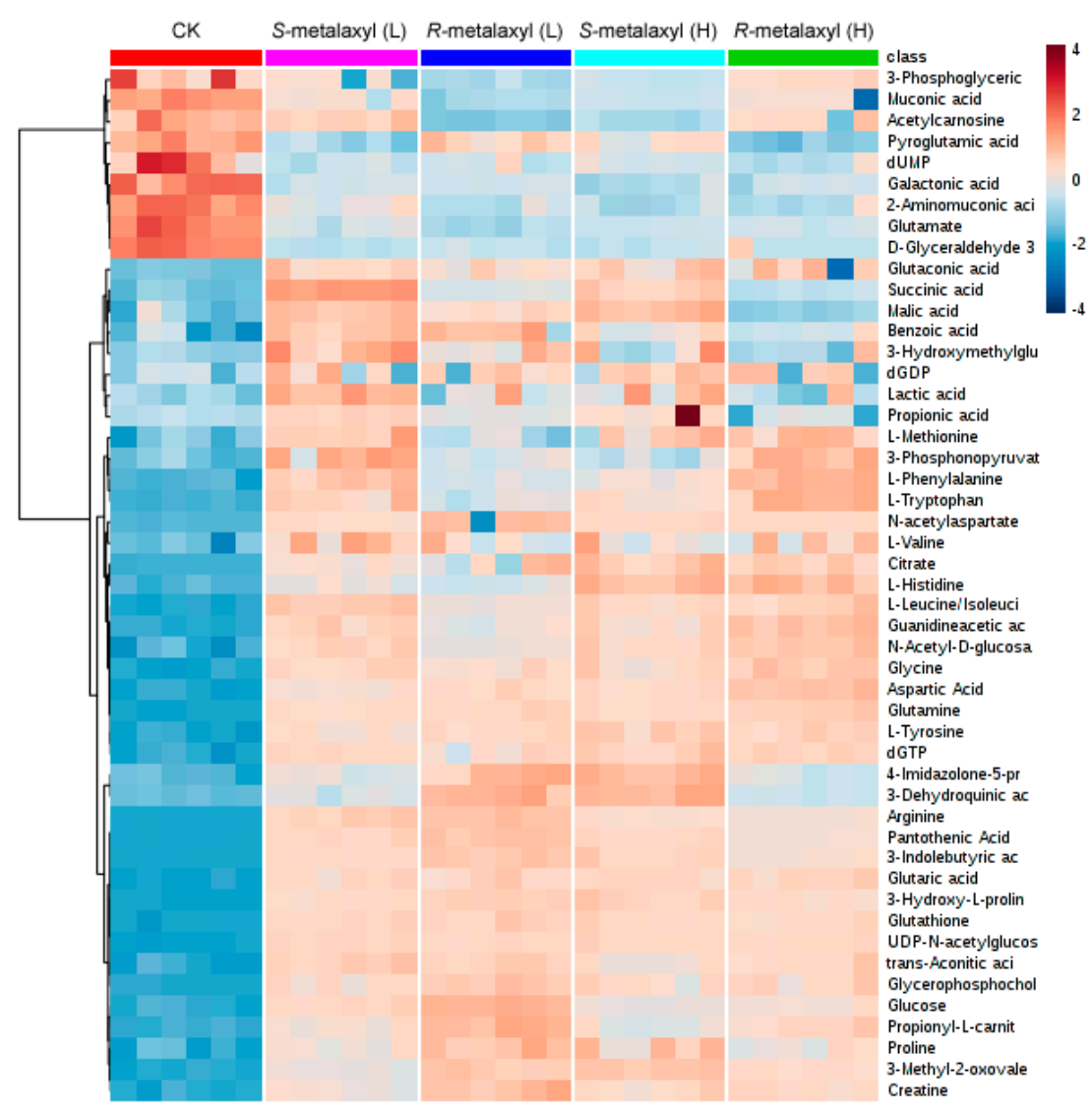

Figure 4. Heat map produced by the most significantly differential metabolites.

\subsection{Biological Pathway Analysis}

Metabolomics profiling can reveal not only the individual metabolite alteration but also provide a comprehensive view of the metabolic processes induced by toxic environmental compounds. In this study, metalaxyl enantiomer-induced metabolic perturbations were further evaluated at the metabolic pathways level on the basis of these different metabolites. The MetaboAnalyst 3.0 was employed to reveal the most significantly affected pathways induced by metalaxyl enantiomers. $R$-metalaxyl mainly affected seven pathways, including Glycine, serine and threonine metabolism, D-Glutamine and D-glutamate metabolism, Glutathione metabolism, Pantothenate and CoA biosynthesis, Arginine and proline metabolism, -TCA cycle- and Phenylalanine metabolism. Whereas, S-metalaxyl mainly caused 10 pathway perturbations, including Glycine, serine and threonine metabolism, D-Glutamine and D-glutamate metabolism, Glutathione metabolism, Alanine, aspartate and glutamate metabolism, Histidine metabolism, Arginine and proline metabolism, Phenylalanine, tyrosine and tryptophan biosynthesis, Pantothenate and CoA biosynthesis, -TCA cycle-and Phenylalanine metabolism. Such results implied that the pathways perturbed by metalaxyl enantiomers were also enantioselective. In addition, Figure 5 indicates that metabolic pathways changes caused by the same concentration of $R$-metalaxyl and $S$-metalaxyl are also enantioselective. Such perturbed pathways are highly related to energy metabolism, amino acid metabolism, lipid metabolism, and antioxidant defense (Figure 6). 

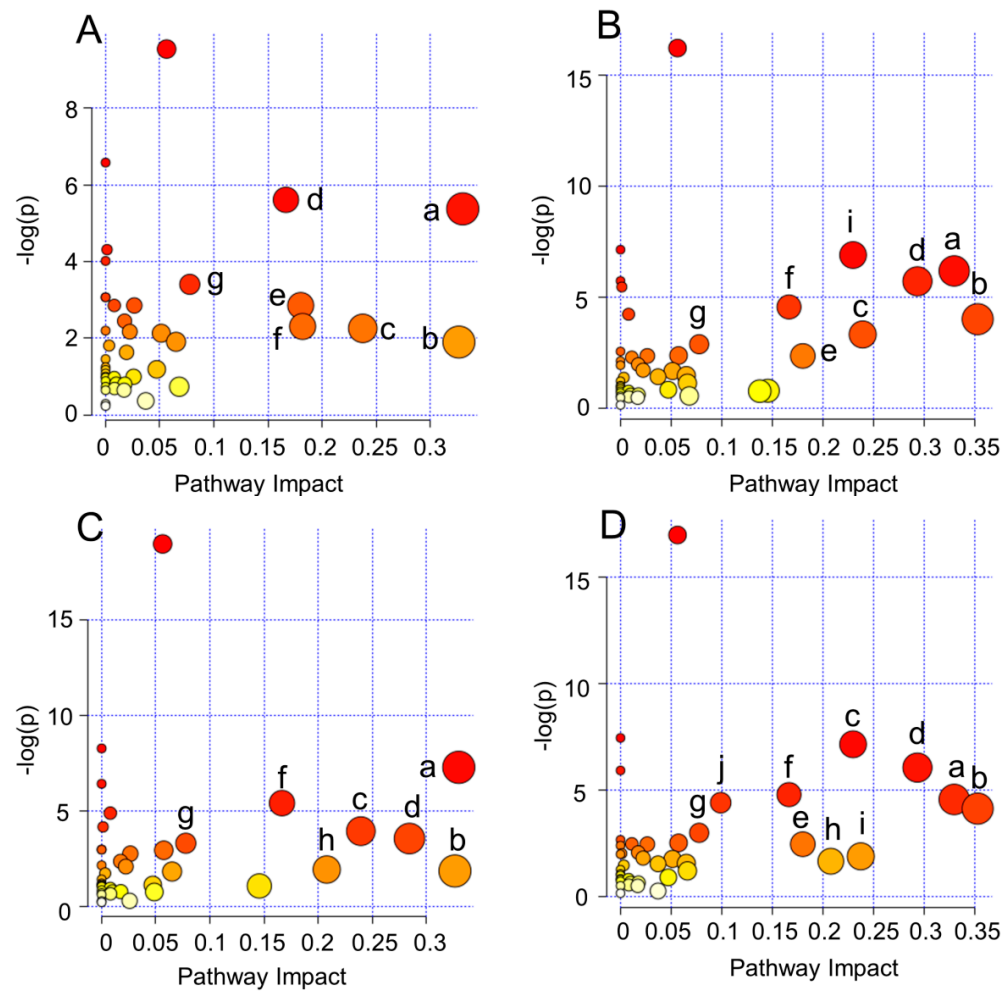

Figure 5. Global metabolic pathways affected by metalaxyl enantiomers based on MetaboAnalyst 3.0. (A) S-metalaxyl (10 $\mu \mathrm{M})$; (B) $R$-metalaxyl (10 $\mu \mathrm{M})$; (C) $S$-metalaxyl (50 $\mu \mathrm{M})$; (D) $R$-metalaxyl $(50 \mu \mathrm{M})$. a. Glycine, serine, and threonine metabolism; b. D-Glutamine and D-glutamate metabolism; c. Glutathione metabolism; d. Arginine and proline metabolism; e. Pantothenate and CoA biosynthesis; f. Phenylalanine metabolism; g. -TCA cycle-; h. Histidine metabolism; i. Alanine, aspartate, and glutamate metabolism; j. Phenylalanine, tyrosine, and tryptophan biosynthesis.

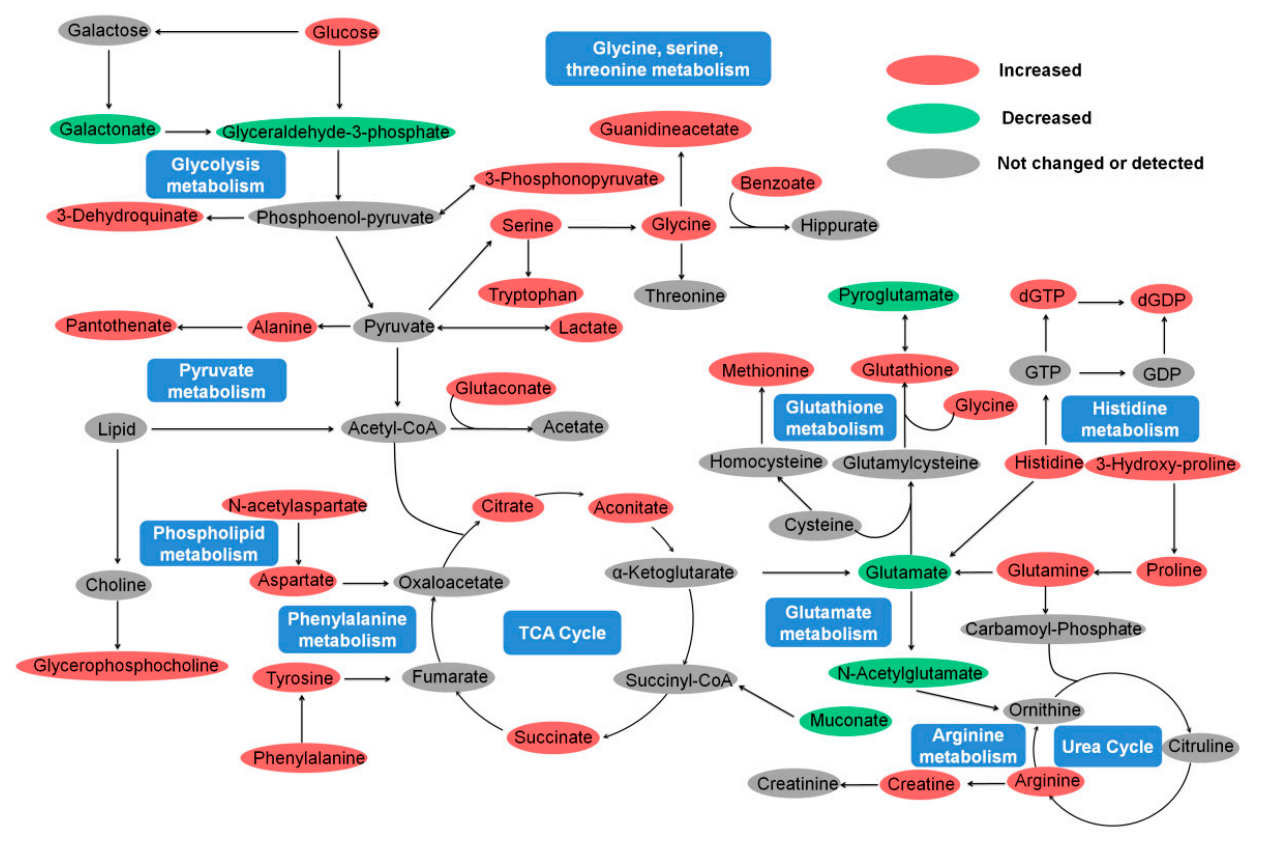

Figure 6. Perturbed pathways and fluctuant metabolites in MCF-7 cells induced by metalaxyl enantiomers. 


\subsection{Amino Acid Metabolism}

Amino acids and their metabolites are critical to life and play a variety of roles in metabolism. Besides their roles as building blocks of polypeptides and proteins, some amino acids including proline, arginine, glutamine, tryptophan, cysteine, and leucine are critical for growth, maintenance, immunity, and reproduction in organisms. Lots of studies also indicate that the abnormal metabolism of amino acids impairs development and growth, perturbs whole body homeostasis, and even causes death. Thus, physiological concentrations of amino acids and their metabolites are relatively constant and highly regulated at the molecular level. In the present study, cell metabolomics results revealed that amino acid metabolism were significantly perturbed due to metalaxyl enantiomers exposure, including Glycine, serine and threonine metabolism, Alanine, aspartate and glutamate metabolism, D-Glutamine and D-glutamate metabolism, Phenylalanine metabolism, Arginine and proline metabolism, Tryrosine metabolism, and so on. Metalaxyl caused increased levels of alanine, tyrosine, phenylalanine, serine, tryptophan, glycine, proline, glutamine, arginine, methionine and decreased levels of glutamate, and $\mathrm{N}$-acetylglutamate. These amino acids regulate specific pathways and have different functions in cell metabolism. Much evidence also shows that amino acids including alanine, glutamine, glutamate, lysine, phenylalanine, and glycine also participate in specific cell metabolism, cell signaling, and oxidative stress [33-35].

Glycine, produced in the mitochondria, has been demonstrated as an indicator of cancer cell proliferation in a previous investigation [36]. In this study, the concentration of glycine increased after metalaxyl enantiomer treatments, which implies that metalaxyl enantiomers may promote MCF-7 cell proliferation. Alanine can regulate gluconeogenesis to ensure glucose production through inhibiting L-type pyruvate kinase [37,38]. Moreover, alanine is also a known product of glucose and glutamine in cancer cells associated with $\beta$-nicotinamide adenine dinucleotide phosphate (NADPH) production. Therefore, increased levels of alanine and glucose may imply upregulated gluconeogenesis and glycolysis in MCF-7 cells after metalaxyl exposure. In cancer cells, glutamine can be utilized for the production of lactate and act as a carbon source and an amino acid for protein synthesis. Once taken up by the cell, much of the glutamine is generally converted to glutamate. It is interesting to point out that metalaxyl enantiomer treatment in MCF-7 cells leads to an increase in glutamine and a decrease in glutamate, which may be caused by upregulated glutamine synthesis and downregulated glutamine metabolism. To sum up, metalaxyl enantiomers can significantly perturb amino acid metabolism in MCF-7 cells in an enantiomer-specific way.

\subsection{Energy Metabolism}

Significant changes of many metabolites involved in energy metabolism, including glucose, lactate, alanine, citrate, aconitate, and succinate, were observed in MCF-7 cells after metalaxyl enantiomer exposure. These metabolites are highly related to energy metabolism pathways such as the TCA cycle, pyruvate metabolism, and glycolysis metabolism. The prominent perturbations of energy metabolism induced by metalaxyl enantiomers were increased levels of TCA cycle intermediates, including citrate, aconitate, and succinate in MCF-7 cells. Citrate, aconitate, and succinate are the main intermediates of the TCA cycle and play important roles in energy metabolism. Although other factors cannot be excluded, the increased levels of citrate, aconitate, and succinate indicated that the activities of mitochondrial enzymes involved in the TCA cycle were significantly affected in MCF-7 cells after metalaxyl enantiomer exposure, resulting in the fluctuation of energy metabolism. Furthermore, the increased levels of citrate and aconitate induced by $R$-metalaxyl and $S$-metalaxyl were almost the same, while succinate increased about 1.2-fold and 1.8-fold after $R$-metalaxyl and $S$-metalaxyl exposure, respectively. This notion suggests that $R$-metalaxyl and $S$-metalaxyl have enantioselective effects on TCA cycle.

In addition, the levels of pantothenate, lactate, and alanine, which are highly related to pyruvate metabolism, were all increased. Pyruvate metabolism has been reported as one of the major alterations in breast cancer cells. Rather than importing pyruvate into mitochondria, cancer cells generally convert 
pyruvate into lactate. The key factor affecting pyruvate metabolism is pyruvate dehydrogenase (PDH), which is highly regulated by pyruvate dehydrogenase kinase (PDK). Inhibition of PDH will decrease transportation of pyruvate into the mitochondria through its oxidation into acetyl-CoA and lead to an increased level of lactate in the cytoplasm [30]. Therefore, the increased level of lactate may be caused by the inhibition of PDH through activating PDK, resulting in decreased transportation of pyruvate into the mitochondria and enhanced conversion of pyruvate to lactate in MCF-7 cells. Furthermore, some gluconeogenic amino acids, including phenylalanine, histidine, methionine, glutamine, tyrosine, and alanine, were all increased, which may imply promoted gluconeogenesis after metalaxyl exposure.

We also found an increased level of creatine in MCF-7 cells after metalaxyl enantiomers exposure. The major function of creatine is to support energy production in the phosphorylation process. Interestingly, studies reported that creatinine and creatine kinase were considered oxidative stress indicators in breast cancer patients after chemotherapy treatment [39]. Therefore, the upregulated level of creatine clearly implies the alteration of oxidative stress and energy metabolism in MCF-7 cells after metalaxyl enantiomer exposure. Taking all these results together, metalaxyl enantiomers significantly perturbed energy metabolism in MCF-7 cells and the degrees affected by two enantiomers were enantioselective.

\subsection{Lipid Metabolism and Antioxidant Defense}

Based on metabolomics results, the upregulated glycerophosphocholine and related amino acids clearly reflected the perturbation of lipid metabolism after metalaxyl enantiomer exposure. Glycerophosphocholine (GPC) is known to be an important endogenous compound required for cell and mitochondrial membranes, neurotransmitter synthesis, methylation-dependent biosynthesis, lipid transportation, and bile acid secretion [40-42]. In addition, GPC is a vital constituent of the cell membrane and lipoprotein phospholipid, which play important roles in the integrity of the cell membrane and lipid metabolism. Evidence shows that GPC metabolite deficiency contributes to various disorders in humans, including fatty liver development, liver steatosis, hepatocarcinogenesis, and mitochondrial dysfunction [43-45]. GPC can not only sustain choline and its metabolites' homeostasis but also protects cells and their organelles from oxidative stress and inflammation [46-49]. In this study, the level of GPC increased after $R$-metalaxyl and $S$-metalaxyl exposure, possibly induced by the high demand of membrane synthesis for cell proliferation after metalaxyl enantiomer exposure.

Another interesting finding is the increased level of glutathione (GSH) after metalaxyl exposure. As the most abundant thiol-containing compound in the cell, GSH plays vital roles in intracellular signaling and antioxidant defense. Total GSH concentration varies significantly in different cell types and can be regulated by external factors including heavy metals, glucose concentration, and exposure to reactive oxygen species. Cancer cells are often protected from excessive damage by reactive oxygen species (ROS) through the simultaneous upregulation of innate protective antioxidant pathways and the generation of reduced glutathione [50]. As a result, the increased GSH induced by metalaxyl enantiomers clearly suggests the enhancement of antioxidant defense in MCF-7 cells, which may be beneficial to promote proliferation of breast cancer cells.

\section{Materials and Methods}

\subsection{Chemicals and Materials}

Racemic metalaxyl (rac-metalaxyl) standard (purity $\geq 98.0 \%$ ) was obtained from the Institute for Control of Agrochemicals, China Ministry of Agriculture (Beijing, China). S-metalaxyl and R-metalaxyl were prepared via high-performance liquid chromatography (HPLC) (Agilent, Santa Clara, CA, USA) with a semi-preparative chiral column containing cellulose-tris-(3,5-methylphenylcarbamate)-based chiral stationary phase (CDMPC-CSP). Water was purified with a Milli-Q Element system from Millipore (Billerica, MA, USA). HPLC grade methanol, acetonitrile, ammonium acetate, and acetic 
acid were purchased from Merck Chemicals (Beijing, China). All other chemicals and solvents were analytical grade and purchased from commercial sources.

\subsection{Cell Culture Procedures and Metabolite Extraction}

The MCF-7 cell line was purchased from the China Center for Type Culture Collection (CCTCC) and cultured in DMEM High Glucose supplemented with $2 \mathrm{mM}$ glutamine, $25 \mathrm{mM}$ glucose, $100 \mathrm{~g} / \mathrm{mL}$ penicillin/streptomycin, and $10 \%$ fetal bovine serum (FBS). Cells were seeded onto $150 \mathrm{~mm}$ plates and the medium was changed every second day until the confluency reached $80 \%$, at which time they were washed with PBS and incubated in DMEM medium containing metalaxyl enantiomers for seven consecutive days. The new medium with metalaxyl enantiomers was also changed every second day. Two concentrations ( 10 and $50 \mu \mathrm{M}$ ) were selected for $S$-metalaxyl and $R$-metalaxyl treatments separately and seven replicate samples were collected for each treatment. After washing the cells with PBS three times, the number of living cells was counted using a Neubauer counting chamber under a light microscope and protein content was analyzed by the kits at the end point. Cold extraction solvent methanol:choloroform (9:1) was added to quench the cellular metabolism. The cells were detached using a cell scraper and the cell suspensions were transferred into tubes and centrifuged. Finally, the supernatants were collected and dried under a vacuum using Eppendorf Concentrator plus (Eppendorf, Germany). Then they were reconstituted in $500 \mu \mathrm{L} 60 \%$ acetonitrile/40\% water solvent containing $5 \mathrm{mM}$ ammonium acetate and $0.2 \%$ acetic acid. All samples were purified with $0.22 \mu \mathrm{m}$ filters before HPLC-QTOF analysis.

\subsection{HPLC-QTOF Analysis}

The HPLC-QTOF analysis was conducted on an Agilent 1200 series HPLC system coupled with an electrospray ionization (ESI) source and Agilent 6510 QTOF mass spectrometry (Agilent, USA). In all cases, $10 \mu \mathrm{L}$ of extracted sample was injected into a reversed-phase column (ACQUITY BEH $\mathrm{C}_{18}, 150 \mathrm{~mm} \times 2.1 \mathrm{~mm}, 1.7 \mu \mathrm{m}$, Waters, Milford, CT, USA) equipped with a guard cartridge system. The flow rate was $0.3 \mathrm{~mL} / \mathrm{min}$ and the column temperature was maintained at $40{ }^{\circ} \mathrm{C}$. The mobile phase was composed of solvents A $\left(10 \%\right.$ acetonitrile $/ 90 \% \mathrm{H}_{2} \mathrm{O}$ containing $5 \mathrm{mM}$ ammonium acetate and $0.2 \%$ acetic acid) and $\mathrm{B}\left(90 \%\right.$ acetonitrile $/ 10 \% \mathrm{H}_{2} \mathrm{O}$ containing $5 \mathrm{mM}$ ammonium acetate and $0.2 \%$ acetic acid). The gradient conditions for both modes were identical and are shown in Table S1 in the Supplementary Materials.

Data were collected in the positive and negative electrospray modes in separate runs with full scan mode ranges from $m / z 60$ to 1000 . The capillary voltages were 3800 and $-4000 \mathrm{~V}$ for positive mode and negative mode, respectively, with a scan rate of 1.03 scans per second. The nebulizer gas flow rate was $10 \mathrm{~L} / \mathrm{min}$; the pressure was maintained at $45 \mathrm{psi}$ and the temperature at $325^{\circ} \mathrm{C}$. Reference masses 121.0509, 922.0098 (positive mode) and 119.0363, 966.0007 (negative mode) were used for continuous and online mass calibration throughout the analyses. All samples were injected in one randomized sequence and kept in the LC auto-sampler maintained at $4{ }^{\circ} \mathrm{C}$.

\subsection{Data Treatment}

The resulting data files were cleaned of unrelated ions and background noise by the Molecular Feature Extractor (MFE) tool in the MassHunter Qualitative Analysis Software (Agilent, USA). The MFE is a compound-finding technique that can extract individual compound features from QTOF-MS chromatogram even when chromatograms are complicated and compounds are not well resolved. Lastly, MFE can output a list of all possible compound features extracted from full scan QTOF data. The MassHunter Mass Profiler Professional Software B.02.00 (Agilent, USA) was used to align and filter off extracted features. We selected metabolites with absolute abundance over 5000 counts and with a minimum of two ions. Metabolites from different samples were aligned using a retention time window of $0.1 \%$ ( $0.15 \mathrm{~min})$ and multiple charge states were not selected. Common features represented in at least $80 \%$ of all samples were analyzed and corrected for individual bias. 


\subsection{Biological Pathway Analysis}

The major perturbed biological pathways were analyzed based on the significantly changed metabolites in MCF-7 cells after metalaxyl enantiomer exposure. In this study, metabolic pathway perturbations were conducted by MetaboAnalyst 3.0 (http://www.metaboanalyst.ca/) and related metabolic pathway profiles were determined based on the Kyoto Encyclopedia of Genes and Genomes (KEGG) pathway database (http://www.kegg.jp/kegg/pathway.html).

\subsection{Statistical Analysis}

Metabolite differences between metalaxyl-treated cell samples and control samples were evaluated using student's $t$ test analysis. Multivariate data analysis was conducted with the SIMCA-P software package (V11.0, Umetrics, Sweden). Principal component analysis (PCA) was conducted based on QTOF datasets to generate an overview for group clustering and to search for possible outliers. Partial least-squares discriminant analysis (PLS-DA) was employed to explore the significant changed metabolites and group clustering based on QTOF datasets. Significantly changed metabolites were identified based on student's $t$ test (with $p<0.05$ ) and variable influence on project score (VIP > 1). Accurate masses of significant changed features were further identified by Mass Profiler Professional Software (Agilent, USA) and also confirmed against HMDB (http://www.hmdb.ca), METLIN (https://metlin.scripps.edu), KEGG (http://www.kegg.jp), and LIPID MAPS (http:/ /www. lipidmaps.org) database.

\section{Conclusions}

In the present study, the enantioselective metabolic perturbations in MCF-7 cells induced by metalaxyl enantiomers were evaluated using HPLC-QTOF-based metabolomics. Our results indicated that metabolic profiles of MCF-7 cells were significantly altered in both metalaxyl enantiomer-treated groups compared with control group. Furthermore, the endogenous metabolite changes and pathway fluctuations induced by metalaxyl enantiomers were enantioselective. HPLC-QTOF-based metabolomics demonstrated that metalaxyl enantiomers mainly disrupted amino acid metabolism, energy metabolism, lipid metabolism, and antioxidant defense. Our study illustrates that QTOF-based metabolomics is very sensitive and suitable for monitoring the systematic metabolic effects of metalaxyl enantiomer exposure in MCF-7 cells, which can provide integrative information for the enantioselective effects of chiral pesticides in breast cancer progression, reveal the underlying mechanisms, and provide available data for the health risk assessment of chiral environmental pollutants at the molecular level.

Supplementary Materials: Supplementary materials can be found at www.mdpi.com/1422-0067/18/1/142/s1.

Acknowledgments: This study was financially supported by the National Natural Science Foundation of China (21207158, 21337005).

Author Contributions: Ping Zhang, Lin He, and Wentao Zhu conceived and designed this study; Ping Zhang, Dezhen Wang, and Jin Yan conducted the experiments; Ping Zhang and Yao Wang analyzed the data; Ping Zhang, Wentao Zhu, Yao Wang, and Lin He prepared the manuscript.

Conflicts of Interest: The authors declare no conflict of interest.

\section{References}

1. Infante-Rivard, C.; Labuda, D.; Krajinovic, M.; Sinnett, D. Risk of childhood leukemia associated with exposure to pesticides and with gene polymorphisms. Epidemiology 1999, 10, 481-487. [CrossRef] [PubMed]

2. Alavanja, M.C.; Hoppin, J.A.; Kamel, F. Health effects of chronic pesticide exposure: Cancer and neurotoxicity. Annu. Rev. Public Health 2004, 25, 155-197. [CrossRef] [PubMed]

3. Blair, A.; Freeman, L.B. Epidemiologic studies in agricultural populations: Observations and future directions. J. Agromed. 2009, 14, 125-131. [CrossRef] [PubMed]

4. EPA (U.S. Environmental Protection Agency). Chemicals Evaluated for Carcinogenic Potential Office of Pesticide Programs; U.S. Environmental Protection Agency: Washington, DC, USA, 2015. 
5. Fryzek, J.P.; Garabrant, D.H.; Harlow, S.D.; Severson, R.K.; Gillespie, B.W.; Schenk, M.; Schottenfeld, D. A case-control study of self-reported exposures to pesticides and pancreas cancer in southeastern Michigan. Int. J. Cancer 1997, 72, 62-67. [CrossRef]

6. Leet, T.; Acquavella, J.; Lynch, C.; Anne, M.; Weiss, N.S.; Vaughan, T.; Checkoway, H. Cancer incidence among alachlor manufacturing workers. Am. J. Ind. Med. 1996, 30, 300-306. [CrossRef]

7. Undeger, U.; Basaran, N. Effects of pesticides on human peripheral lymphocytes in vitro: Induction of DNA damage. Arch. Toxicol. 2005, 79, 169-176. [CrossRef] [PubMed]

8. Hooghe, R.J.; Devos, S.; Hooghe-Peters, E.L. Effects of selected herbicides on cytokine production in vitro. Life Sci. 2000, 66, 2519-2525. [CrossRef]

9. Corsini, E.; Codeca, I.; Mangiaratti, S.; Birindelli, S.; Minoia, C.; Turci, R.; Viviani, B.; Facchi, A.; Vitelli, N.; Lucchi, L.; et al. Immunomodulatory effects of the herbicide propanil on cytokine production in humans: In vivo and in vitro exposure. Toxicol. Appl. Pharmacol. 2007, 222, 202-210. [CrossRef] [PubMed]

10. Stiller-Winkler, R.; Hadnagy, W.; Leng, G.; Straube, E.; Idel, H. Immunological parameters in humans exposed to pesticides in the agricultural environment. Toxicol. Lett. 1999, 107, 219-224. [CrossRef]

11. Buser, H.-R.; Muller, M.D.; Poiger, T.; Balmer, M.E. Environmental behavior of the chiral acetamide pesticide metalaxyl:enantioselective degradation and chiral stability in soil. Environ. Sci. Technol. 2001, 36, 221-226. [CrossRef]

12. Buerge, I.J.; Poiger, T.; Muller, M.D.; Buser, H.R. Enantioselective degradation of metalaxyl in soils: Chiral preference changes with soil pH. Environ. Sci. Technol. 2003, 37, 2668-2674. [CrossRef] [PubMed]

13. Lu, H. Stereoselectivity in drug metabolism. Expert Opin. Drug Metab. Toxicol. 2007, 3, 149-158. [CrossRef] [PubMed]

14. Hrelia, P.; Maffei, F.; Fimognari, C.; Vigagni, F.; Cantelli-Forti, G. Cytogenetic effects of Metalaxyl on human and animal chromosomes. Mutat. Res. 1996, 369, 81-86. [CrossRef]

15. Paolini, M.; Mesirca, R.; Pozzetti, L.; Sapone, A.; Cantelli-Forti, G. Biomarkers of effect in evaluating metalaxyl cocarcinogenesis. Selective induction of murine CYP 3A isoform. Mutat. Res. 1996, 361, 157-164. [CrossRef]

16. Sakr, S.A.; Badawy, G.M. Effect of ginger (Zingiber officinale R.) on metiram-inhibited spermatogenesis and induced apoptosis in albino mice. J. Appl. Pharm. Sci. 2011, 1, 131-136.

17. DeSantis, C.; Ma, J.; Bryan, L.; Jemal, A. Breast cancer statistics, 2013. CA Cancer J. Clin. 2014, 64, 52-62. [CrossRef] [PubMed]

18. Siegel, R.L.; Miller, K.D.; Jemal, A. Cancer statistics, 2016. CA Cancer J. Clin. 2016, 66, 7-30. [CrossRef] [PubMed]

19. Calle, E.E.; Frumkin, H.; Henley, S.J.; Savitz, D.A.; Thun, M.J. Organochlorines and breast cancer risk. CA Cancer J. Clin. 2002, 52, 301-309. [CrossRef] [PubMed]

20. Ewence, A.; Brescia, S.; Johnson, I.; Rumsby, P.C. An approach to the identification and regulation of endocrine disrupting pesticides. Food Chem. Toxicol. 2015, 78, 214-220. [CrossRef] [PubMed]

21. Marx-Stoelting, P.; Niemann, L.; Ritz, V.; Ulbrich, B.; Gall, A.; Hirsch-Ernst, K.I.; Pfeil, R.; Solecki, R. Assessment of three approaches for regulatory decision making on pesticides with endocrine disrupting properties. Regul. Toxicol. Pharmacol. 2014, 70, 590-604. [CrossRef] [PubMed]

22. Perocco, P.; Colacci, A.; Bonora, B.; Grilli, S. In vitro transforming effect of the fungicides metalaxyl and zineb. Teratog. Carcinog. Mutagen. 1995, 15, 73-80. [CrossRef] [PubMed]

23. Kojima, H.; Takeuchi, S.; Nagai, T. Endocrine-disrupting potential of pesticides via nuclear receptors and aryl hydrocarbon receptor. J. Health Sci. 2010, 56, 374-386. [CrossRef]

24. Chen, Y.; Tang, Y.; Chen, S.; Nie, D. Regulation of drug resistance by human pregnane X receptor in breast cancer. Cancer Biol. Ther. 2009, 8, 1265-1272. [CrossRef] [PubMed]

25. Zhao, X.J.; Hao, F.; Huang, C.; Rantalainen, M.; Lei, H.; Tang, H.; Wang, Y. Systems responses of rats to mequindox revealed by metabolic and transcriptomic profiling. J. Proteome Res. 2012, 11, 4712-4721. [CrossRef] [PubMed]

26. Nicholson, J.K.; Lindon, J.C. Systems biology: Metabonomics. Nature 2008, 455, 1054-1056. [CrossRef] [PubMed]

27. Nicholson, J.K.; Lindon, J.C.; Holmes, E. 'Metabonomics': Understanding the metabolic responses of living systems to pathophysiological stimuli via multivariate statistical analysis of biological NMR spectroscopic data. Xenobiotica 1999, 29, 1181-1189. [CrossRef] [PubMed] 
28. Gatenby, R.A.; Gillies, R.J. Why do cancers have high aerobic glycolysis? Nat. Rev. Cancer 2004, 4, 891-899. [CrossRef] [PubMed]

29. Cairns, R.A.; Harris, I.S.; Mak, T.W. Regulation of cancer cell metabolism. Nat. Rev. Cancer 2011, 11, 85-95. [CrossRef] [PubMed]

30. Lefort, N.; Brown, A.; Lloyd, V.; Ouellette, R.; Touaibia, M.; Culf, A.S.; Cuperlovic-Culf, M. ${ }^{1} \mathrm{H}$ NMR metabolomics analysis of the effect of dichloroacetate and allopurinol on breast cancers. J. Pharm. Biomed. Anal. 2014, 93, 77-85. [CrossRef] [PubMed]

31. Oakman, C.; Tenori, L.; Biganzoli, L.; Santarpia, L.; Cappadona, S.; Luchinat, C.; di Leo, A. Uncovering the metabolomic fingerprint of breast cancer. Int. J. Biochem. Cell Biol. 2011, 43, 1010-1020. [CrossRef] [PubMed]

32. McCombie, G.; Browning, L.M.; Titman, C.M.; Song, M.; Shockcor, J.; Jebb, S.A.; Griffin, J.L. $\omega-3$ oil intake during weight loss in obese women results in remodelling of plasma triglyceride and fatty acids. Metabolomics 2009, 5, 363-374. [CrossRef] [PubMed]

33. Marc Rhoads, J.; Wu, G. Glutamine, arginine, and leucine signaling in the intestine. Amino Acids 2009, 37, 111-122. [CrossRef] [PubMed]

34. Jobgen, W.S.; Fried, S.K.; Fu, W.J.; Meininger, C.J.; Wu, G. Regulatory role for the arginine-nitric oxide pathway in metabolism of energy substrates. J. Nutr. Biochem. 2006, 17, 571-588. [CrossRef] [PubMed]

35. Mannick, J.B. Regulation of apoptosis by protein S-nitrosylation. Amino Acids 2007, 32, 523-526. [CrossRef] [PubMed]

36. Jain, M.; Nilsson, R.; Sharma, S.; Madhusudhan, N.; Kitami, T.; Souza, A.L.; Kafri, R.; Kirschner, M.W.; Clish, C.B.; Mootha, V.K. Metabolite profiling identifies a key role for glycine in rapid cancer cell proliferation. Science 2012, 336, 1040-1044. [CrossRef] [PubMed]

37. Meijer, A.J. Amino acids as regulators and components of nonproteinogenic pathways. J. Nutr. 2003, 133, 2057S-2062S. [PubMed]

38. Xu, J.; Jiang, H.; Li, J.; Cheng, K.K.; Dong, J.; Chen, Z. ${ }^{1} \mathrm{H}$ NMR-based metabolomics investigation of copper-laden rat: A model of Wilson's disease. PLoS ONE 2015, 10, e0119654. [CrossRef] [PubMed]

39. Amin, K.A.; Mohamed, B.M.; El-Wakil, M.A.; Ibrahem, S.O. Impact of breast cancer and combination chemotherapy on oxidative stress, hepatic and cardiac markers. J. Breast Cancer 2012, 15, 306-312. [CrossRef] [PubMed]

40. Kohlmeier, M.; da Costa, K.A.; Fischer, L.M.; Zeisel, S.H. Genetic variation of folate-mediated one-carbon transfer pathway predicts susceptibility to choline deficiency in humans. Proc. Natl. Acad. Sci. USA 2005, 102, 16025-16030. [CrossRef] [PubMed]

41. Al Rajabi, A.; Castro, G.S.; da Silva, R.P.; Nelson, R.C.; Thiesen, A.; Vannucchi, H.; Vine, D.F.; Proctor, S.D.; Field, C.J.; Curtis, J.M.; et al. Choline supplementation protects against liver damage by normalizing cholesterol metabolism in Pemt/Ldlr knockout mice fed a high-fat diet. J. Nutr. 2014, 144, 252-257. [CrossRef] [PubMed]

42. Trousil, S.; Lee, P.; Pinato, D.J.; Ellis, J.K.; Dina, R.; Aboagye, E.O.; Keun, H.C.; Sharma, R. Alterations of choline phospholipid metabolism in endometrial cancer are caused by choline kinase $\alpha$ overexpression and a hyperactivated deacylation pathway. Cancer Res. 2014, 74, 6867-6877. [CrossRef] [PubMed]

43. Zhu, J.; Wu, Y.; Tang, Q.; Leng, Y.; Cai, W. The effects of choline on hepatic lipid metabolism, mitochondrial function and antioxidative status in human hepatic C3A cells exposed to excessive energy substrates. Nutrients 2014, 6, 2552-2571. [CrossRef] [PubMed]

44. Li, Z.; Agellon, L.B.; Vance, D.E. Phosphatidylcholine homeostasis and liver failure. J. Biol. Chem. 2005, 280, 37798-37802. [CrossRef] [PubMed]

45. Zeisel, S.H. Nutritional genomics: Defining the dietary requirement and effects of choline. J. Nutr. 2011, 141, 531-534. [CrossRef] [PubMed]

46. Zhang, L.; Wang, Y.; Xu, Y.; Lei, H.; Zhao, Y.; Li, H.; Lin, X.; Chen, G.; Tang, H. Metabonomic analysis reveals efficient ameliorating effects of acupoint stimulations on the menopause-caused alterations in mammalian metabolism. Sci. Rep. 2014, 4, 3641. [CrossRef] [PubMed]

47. Zhang, L.; Hatzakis, E.; Nichols, R.G.; Hao, R.; Correll, J.; Smith, P.B.; Chiaro, C.R.; Perdew, G.H.; Patterson, A.D. Metabolomics reveals that aryl hydrocarbon receptor activation by environmental chemicals induces systemic metabolic dysfunction in mice. Environ. Sci. Technol. 2015, 49, 8067-8077. [CrossRef] [PubMed] 
48. Bollard, M.E.; Contel, N.R.; Ebbels, T.M.; Smith, L.; Beckonert, O.; Cantor, G.H.; Lehman-McKeeman, L.; Holmes, E.C.; Lindon, J.C.; Nicholson, J.K.; et al. NMR-based metabolic profiling identifies biomarkers of liver regeneration following partial hepatectomy in the rat. J. Proteome Res. 2010, 9, 59-69. [CrossRef] [PubMed]

49. Zhang, L.; Ye, Y.; An, Y.; Tian, Y.; Wang, Y.; Tang, H. Systems responses of rats to aflatoxin B1 exposure revealed with metabonomic changes in multiple biological matrices. J. Proteome Res. 2011, 10, 614-623. [CrossRef] [PubMed]

50. Shanware, N.P.; Mullen, A.R.; DeBerardinis, R.J.; Abraham, R.T. Glutamine: Pleiotropic roles in tumor growth and stress resistance. J. Mol. Med. 2011, 89, 229-236. [CrossRef] [PubMed] 